El efecto de los procesos escolares en el rendimiento en Matemática y las brechas de rendimiento debido a diferencias socioeconómicas de los estudiantes peruanos

\author{
The effect of educational processes \\ on math achievement and achievement gaps \\ due to socioeconomic differences \\ in Peruvian students
}

\author{
Juan León \\ Grupo de Análisis para el Desarrollo \\ jleon@grade.org.pe \\ Min-Jong Youn \\ National Youth Policy Institute \\ myoun@nypi.re.kr
}

Recibido: 28-3-2016

Aprobado: 30-6-2016 


\title{
Resumen
}

Evaluaciones internacionales, regionales y nacionales han mostrado que los estudiantes peruanos tienen serias deficiencias en el aprendizaje de las matemáticas. Así, el identificar variables de procesos escolares que podrían ayudar a mejorar los resultados educativos, en términos de calidad y equidad, es fundamental para la mejora de la educación en nuestro país. El presente estudio se sirve de las bases de datos de Pisa 2012 para estimar un modelo multinivel de coeficientes aleatorios que permita no solo modelar el rendimiento de los estudiantes, sino las brechas educativas asociadas a diferencias socioeconómicas. Los principales resultados del estudio muestran que, entre las variables de procesos escolares usadas en el presente estudio, el clima disciplinario en clase tiene un efecto positivo y significativo sobre el rendimiento promedio de los estudiantes, mientras que el clima escolar y el sentido de pertenencia promedio de los estudiantes en la escuela tienen un efecto sobre las brechas de rendimiento debido a diferencias socioeconómicas.

Palabras clave: procesos escolares, diferencias socioeconómicas, calidad educativa, equidad educativa, Pisa, Perú

\begin{abstract}
International, regional and national evaluations have shown that Peruvian students have serious learning deficiencies in math. Thus, in order to improve Peruvian education, it is key to identify variables of school processes that could ameliorate the educational results in terms of educational quality and educational equity. Using 2012 PISA datasets, we estimate a HLM with Random Coefficients in order to model the effect on math achievement and the achievement gap due to socioeconomic differences. Our results show that, among the variables of school processes used in the present study, a disciplinary climate in class has a positive and significant effect on math achievement, while an adequate school atmosphere and the students' sense of belonging have an effect on achievement gaps due to socioeconomic differences.
\end{abstract}

Keywords: educational processes, socioeconomic differences, educational quality, educational equity, PISA, Peru 


\section{El efecto de los procesos escolares en el rendimiento promedio y las brechas debido a diferencias socioeconómicas de los estudiantes peruanos}

\section{Introducción}

El objetivo del presente estudio es contribuir al conocimiento sobre el efecto que tienen los procesos escolares, y en particular las prácticas pedagógicas, en el rendimiento de los estudiantes peruanos. Si bien, en los últimos años, se han llevado a cabo diferentes estudios relacionados con esta temática, la presente investigación centra su análisis en las variables de procesos escolares asociadas con las brechas de rendimiento debido a diferencias socioeconómicas de los estudiantes peruanos.

Los procesos escolares implican acciones llevadas a cabo en el aula o la institución educativa (IE) para promover el aprendizaje de los estudiantes (Branch \& Deissler, 2013). Su existencia demarca diferencias en contextos de desigualdad social; por ello, ha sido uno de los principales planteamientos del movimiento de investigación en efectividad escolar. Entender cómo operan los procesos escolares tiene relevancia científica y también instrumental, pues se trata de variables que pueden ser modificadas a través de políticas educativas e intervenciones específicas que promuevan los aprendizajes dentro de las instituciones educativas.

En el caso peruano, diferentes estudios han encontrado efectos positivos y significativos de los procesos escolares en el rendimiento de los estudiantes (Banco Mundial, 1999; UMC \& Grade, 2001; Cueto, Ramírez, León \& Guerrero, 2004; Caro, 2004; Asmad et al., 2004; Valdivia, 2004; Agüero \& Cueto, 2004; Miranda, 2008; Cueto et al., 2014). Un ejemplo de ello son los estudios llevados a cabo por Cueto et al. (2004); Cueto, Ramírez y León (2006); y Cueto et al. (2014), en los que se encuentra que la demanda cognitiva de los ejercicios que desarrollan los estudiantes en el aula de clase y la retroalimentación que brindan los docentes tienen un efecto positivo y significativo en su rendimiento. De igual forma, los informes de resultados del primer y del segundo Estudio Regional de Calidad Educativa del Laboratorio Latinoamericano de Calidad Educativa (Llece) muestran que el clima del aula y el sistema de evaluaciones que usan los docentes están asociados con el desempeño de los estudiantes en Comunicación y Matemática (Llece, 2001, 2010). A pesar de lo anterior, ningún estudio ha explorado el efecto de las variables de procesos escolares en la reducción de las brechas de rendimiento de los estudiantes debido a diferencias socioeconómicas, lo cual es pertinente, porque tal información podría tener efectos ecualizadores frente a las inequidades educativas.

El presente estudio busca contribuir en esa dirección. De ese modo, haciendo uso de los modelos multinivel de coeficientes aleatorios, se va a modelar las brechas de rendimiento de los estudiantes debido a diferencias socioeconómicas en escuelas públicas. Aquello permitirá identificar qué variables de procesos escolares ayudan a mejorar la equidad de los resultados educativos de los estudiantes peruanos. La razón por la cual se exploran los efectos sobre las diferencias socioeconómicas en el rendimiento radica en que estudios recientes 
han mostrado que estas son una de las más marcadas entre los factores sociodemográficos de los estudiantes tanto de primaria como secundaria (Benavides, León \& Etesse, 2014; Guadalupe, León \& Cueto, 2013); incluso, en el caso peruano, las diferencias entre el primer y el quinto quintil socioeconómico son de las más acentuadas entre los países que participaron de Pisa en los años 2009 y 2012 (Organización para la Cooperación y el Desarrollo Económico [OECD], 2010, 2013).

El presente informe está dividido en seis secciones. La primera corresponde a esta introducción. La segunda sección presenta el marco teórico que orienta la investigación. La tercera sección describe las preguntas e hipótesis de la investigación. Luego, la cuarta sección detalla la metodología empleada para los análisis y, en la quinta sección, se exponen los principales resultados. Finalmente, en la última sección, se ofrece algunas conclusiones en función a los resultados obtenidos.

\section{Marco teórico}

A mediados de los 60 e inicios de los 70, se llevaron a cabo 2 estudios acerca de las desigualdades educativas en los Estados Unidos, los cuales iniciaron la discusión sobre el rol que cumplen las escuelas y los docentes en el rendimiento de los estudiantes. El primero de estos, realizado por Coleman et al. (1966), tuvo como principal hallazgo que los insumos escolares, como la infraestructura educativa, no tenían un efecto significativo sobre el rendimiento de los estudiantes. Sin embargo, características de los niños y niñas (ej. raza) y sus familias (ej. nivel socioeconómico) tenían un efecto significativo y explicaban la variabilidad de los resultados en rendimiento. Posteriormente, Jencks et al. (1972), usando la misma base de datos, encontraron los mismos resultados sobre la poca relevancia de los insumos escolares. Por otro lado, en Inglaterra, Plowden Committee (1967) dio a conocer la situación de las escuelas primarias, en un estudio en el que se presentó cómo las características de las familias y sus estudiantes tienen un mayor peso en el rendimiento de los estudiantes que las variables escolares.

Estos estudios no solo originaron la discusión acerca del rol de las escuelas y los docentes, sino también el surgimiento de tres corrientes de investigación orientadas a estudiar qué variables escolares ${ }^{1}$ están asociadas a un mayor rendimiento en los estudiantes. Con el fin de ser objeto de políticas educativas, estas corrientes se basan en diferentes modelos conceptuales sobre el proceso educativo que se desarrolla en las escuelas ${ }^{2}$ : i) el modelo de función

1. Las variables escolares implican todas aquellas características vinculadas con la institución educativa, y el aula, que influyen, de manera premeditada o no premeditada, en los procesos de aprendizaje del estudiante. De esta forma, las variables escolares pueden ser conformadas por variables contextuales (ej. insumos de la escuela, infraestructura), prácticas docentes (ej. retroalimentación del docente, clima disciplinario) y procesos escolares (ej. sentido de pertenencia, buena relación docentealumno) (Branch \& Deissler, 2013).

2. Esta taxonomía fue tomada de Schereens y Bosker (1997). 
de producción (Hanushek, 1986; Greenwald, Heges \& Laine, 1996), ii) el modelo de productividad educativa (Walberg, 1984) y iii) modelos de efectividad integrada (Scheerens, 1990; Creemers, 1994; Stringfield \& Slavin, 1992).

El modelo de función de producción se aproxima al proceso educativo como una relación de insumo-producto, en la que los insumos son las características de las escuelas y sus docentes, y el producto son los resultados cognitivos de los estudiantes. Hanushek (1986), y Greenwald et al. (1996) llevaron a cabo una revisión sistemática de los estudios que exploran las relaciones entre insumos escolares y rendimiento. En este, encontraron que existen características de las escuelas y de los docentes asociadas de manera significativa con el rendimiento de los estudiantes. De esta forma, concluyen que es necesario descartar el pensamiento de que la inversión en insumos escolares no tiene efecto sobre los resultados de los estudiantes.

El segundo modelo de productividad educativa es similar al de función de producción, pero con dos diferencias principales: i) la inclusión de variables relacionadas a procesos de aprendizaje y ii) el tomar como unidad de análisis al docente. Walberg (1984) plantea un primer modelo de efectividad educativa, que se basa en el modelo de aprendizajes escolares de Carroll (1963) y lo expande. Además de las cinco variables fundamentales consideradas por Carroll (1963) -habilidad, madurez, motivación, oportunidades de aprender y calidad de instrucción-, Walberg (1984) incluye variables del contexto que influyen en los resultados educativos, tales como características del hogar, el docente, sus pares y uso del tiempo fuera de la escuela. De esta manera, plantea que el proceso educativo no es solo determinado por variables del individuo, sino también por variables relacionadas con la instrucción y el contexto educativo.

Finalmente, el tercer modelo conceptual es desarrollado a inicios de los 90. En esa línea, se encuentran los estudios realizados por Scheerens (1990) y Creemers (1994) en Europa, y Stringfield y Slavin (1992) en Estados Unidos. El modelo recoge las ideas de los modelos anteriores y adiciona los siguientes aspectos: i) la inclusión de variables de procesos escolares en la escuela y ii) la interacción entre niveles. En este segundo aspecto, se analiza si las variables escolares (insumos, contexto o procesos) impactan en la relación entre variables del docente y el rendimiento del estudiante, así como si las variables a nivel del docente impactan en la relación entre las variables del estudiante y el rendimiento. Gracias a estas ideas, el modelo puede explorar dos aspectos principales. En primer lugar, es posible evaluar qué variables de los procesos escolares permiten mejorar el desempeño de los estudiantes. En segundo lugar, a partir de este, se pueden explorar las interacciones entre niveles que permiten que se modelen las brechas de rendimiento debido a diferentes aspectos sociales y demográficos de los estudiantes.

Las tres corrientes detalladas se encuentran incluidas dentro de la literatura educativa sobre efectos escolares ${ }^{3}$, pero solo las dos últimas pueden ser tomadas

3. En la literatura internacional, se denomina «School effects». 
en cuenta dentro de los modelos de eficacia escolar. Ello se debe a que exploran el efecto de variables de procesos escolares sobre el rendimiento de los estudiantes controlando las diferencias socioeconómicas y demográficas. De este modo, el enfoque de efectividad integrada se constituye como el más completo, puesto que permite estimar los efectos de los procesos escolares sobre el desempeño de los estudiantes y, a partir de ello, explorar aspectos de la calidad educativa.

Partiendo de todo lo anterior, es pertinente definir el concepto de procesos escolares a partir de la literatura de eficacia escolar. Branch y Deissler (2013) nos indican que son las diferentes acciones que realizan los actores educativos, es decir, estudiantes, docentes y directores. Estas acciones, dirigidas tanto a nivel del aula como de la institución educativa, tienen como resultado final la promoción del aprendizaje de los estudiantes. Así mismo, las variables de procesos escolares identificadas por los estudios de eficacia escolar son resumidos por Luyten, Scheerens y Van Ravens (2011) en tres niveles: i) sistema, que incluye los aspectos macro vinculados a lineamientos políticos generales, la malla curricular y el financiamiento de las escuelas; ii) institución educativa, que implica aquellas gestiones al interior de las escuelas, a nivel de infraestructura y pedagogía; y iii) aula de clase, que resalta las dinámicas entre docentes y estudiantes que cotidianamente influyen en los procesos de aprendizaje de los segundos.

En los últimos años, son diversos los estudios que han explorado el efecto de las variables escolares en el desempeño de los estudiantes, ya sea a nivel internacional (Luyten et al., 2011) o regional (Murillo et al., 2007). Sin embargo, son pocos los estudios que han explorado el efecto de las variables escolares sobre las brechas de rendimiento que responden a diferencias socioeconómicas de los estudiantes. Lee y Bryk (1989) desarrollaron uno de los primeros estudios con esta temática y, encontraron que, para una muestra de estudiantes de secundaria, un clima escolar adecuado ayudaba a reducir las brechas de rendimiento en Matemáticas debido a diferencias por raza; por su parte, el tamaño de la escuela y el ambiente disciplinario ayudan a reducir las brechas de rendimiento debido a clases sociales y antecedentes académicos.

Lee, Smith y Croninger (1997) prestaron atención a las variables relacionadas con los procesos escolares. En una muestra de estudiantes de secundaria en Estados Unidos, hallaron que los procesos vinculados a la responsabilidad colectiva y organización académica de la escuela contribuyen a reducir las brechas de rendimiento en Ciencias y Matemáticas generadas por diferencias socioeconómicas de los estudiantes. De otra forma, los hallazgos de Albernaz, Ferreira y Franco (2002) en escuelas secundarias de Brasil sugieren que el nivel educativo de los docentes y las variables de eficiencia interna de las escuelas ayudan a explicar las brechas de rendimiento debido a diferencias socioeconómicas.

Asimismo, Franco et al. (2007) exploraron esta temática en una muestra de estudiantes de primaria. Los investigadores encontraron que las brechas de rendimiento debido a diferencias socioeconómicas incrementaban en escuelas 154 I donde los docentes dan retroalimentación a los estudiantes y trabajan temas 
de resolución de problemas en clase. Este resultado, a diferencia de los anteriores, muestra que aquellos procesos escolares tienen una magnitud diferente por grupos socioeconómicos: los de mayor nivel socioeconómico son los que reciben en mayor cantidad este tipo de prácticas; por tanto, se ampliaría más las brechas de rendimiento por diferencias socioeconómicas.

Finalmente, Lee, Franco y Albernaz (2009) realizan un estudio comparativo en cinco países con la finalidad de ver el efecto de diferentes variables escolares relacionadas a políticas educativas sobre el rendimiento promedio de los estudiantes y las brechas de rendimiento debido a diferencias socioeconómicas. Los autores, haciendo uso de las bases de datos del estudio Pisa 2003, no encontraron variables transversales que permitan afirmar que existe una política educativa universal para reducir la inequidad en las escuelas; sin embargo, hallaron que las tasas de ausentismo de los estudiantes y el sentido de pertenencia ayudan a reducir las brechas de rendimiento por niveles socioeconómicos en Brasil y México, respectivamente.

El mapa conceptual mostrado a continuación es una adaptación del mapa propuesto por Scheerens (1990) y resume la información acerca de las diferentes relaciones planteadas en esta sección.

Figura 1. Mapa conceptual sobre eficacia escolar

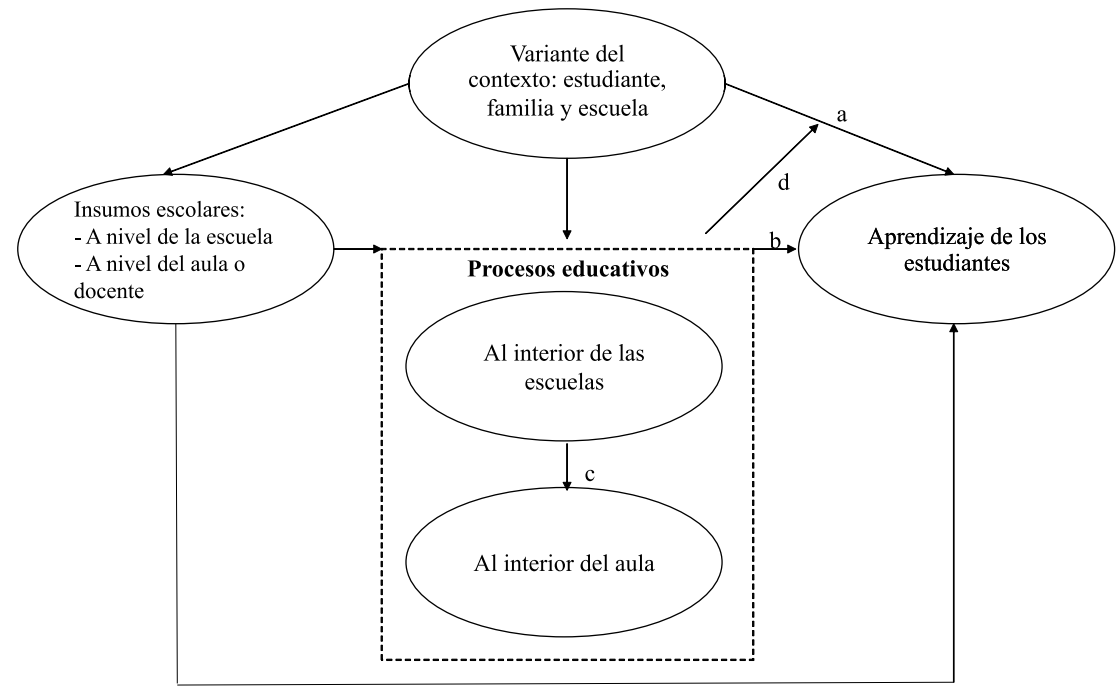

$\mathrm{a}=$ El efecto de las variables de contexto de los estudiantes y sus familias sobre el rendimiento (ej. nivel socioeconómico)

$\mathrm{b}=$ El efecto de las variables de procesos escolares sobre el rendimiento

$c=$ Interacción entre variables de procesos escolares de la escuela y del aula

$\mathrm{d}=$ El efecto de las variables de procesos escolares sobre la brecha socioeconómica de los estudiantes y su familia

Fuente: Elaboración propia 


\subsection{Eficacia escolar en Latinoamérica}

La literatura acerca de la eficacia escolar en Latinoamérica no es abundante, pero existe un número considerable de estudios sobre el tema que se han venido realizando durante las últimas dos décadas, tanto de corte cualitativo y cuantitativo (Murillo, 2003). Los estudios de corte cualitativo indican que las variables de procesos escolares son importantes y estarían explicando los buenos resultados de las escuelas consideradas como efectivas. Los resultados más resaltantes son la coordinación de prácticas de enseñanza entre docentes, la buena relación entre los diferentes actores educativos en la escuela, el acompañamiento pedagógico en el desarrollo de los aprendizajes, la organización de la escuela y el liderazgo de las autoridades de la escuela.

Los estudios cuantitativos son los que, en mayor medida, se han realizado en las últimas dos décadas. Este predominio se debe, principalmente, a que desde 1990 se ha incrementado el número de bases de datos disponibles para el desarrollo de investigación educativa. Este incremento en la disponibilidad de la información ha sido motivado por tres factores. En primer lugar, destaca la mayor participación de países latinoamericanos en evaluaciones internacionales, como el Programme for International Student Assessment (Pisa), Trends of International Mathematics and Science Study (Timss) y Progress of International Reading Literacy Study (Pirls). En segundo lugar, se debe considerar el desarrollo de evaluaciones regionales en Latinoamérica como el primer y el segundo Estudio Regional Comparativo Educativo por parte del Laboratorio Latinoamericano de Calidad Educativa (Llece) en 1997 y 2006, respectivamente, y el estudio de eficacia escolar en Iberoamérica, realizado por el Convenio Andrés Bello (Cab) en el año 2002 (Murillo et al., 2007). En tercer lugar, se encuentra el continuo desarrollo de evaluaciones nacionales del rendimiento al interior de cada país en la región. Finalmente, se debe tomar en cuenta el mayor desarrollo de estudios de casos al interior de cada país para describir y entender la problemática escolar.

Los principales estudios de corte cuantitativo son los estudios realizados a nivel regional por el Laboratorio Latinoamericano de Evaluación de la Calidad de la Educación (Llece) en los años 1997 (Llece, 2001) y 2006 (Llece, 2010) y por la Cab en el 2002 (Murillo et al., 2007). Estos estudios, además de comparar el rendimiento de los estudiantes en Latinoamérica, permitieron explorar qué variables de procesos escolares están asociadas con un mayor rendimiento en Matemática y Comprensión Lectora. Así, en los estudios realizados por Llece (2001, 2010), se encontró que el clima escolar permite explicar las diferencias en el rendimiento escolar incluso cuando se controla por el nivel socioeconómico de los estudiantes y los insumos de las escuelas. Otras variables de procesos significativas, pero solo en el primer estudio, fueron la evaluación continua de los estudiantes y la participación activa de los padres en la escuela. En el caso del estudio realizado por la Cab, a diferencia del que llevó a cabo Llece, la muestra intencional de escuelas está conformada por nueve países de 
Iberoamérica, que fueron seleccionados de acuerdo con el nivel de desempeño de sus estudiantes ${ }^{4}$. Este estudio encuentra que las variables de procesos asociadas con el rendimiento en Comprensión Lectora y Matemática son el clima en el aula, la preparación de las clases por parte de los docentes, satisfacción con el trabajo en la escuela, tiempo de enseñanza y la calidad del currículo.

A nivel de cada país en la región, diferentes estudios han abordado el análisis de los procesos escolares al interior de las aulas de clase y su efecto sobre el rendimiento. Estos estudios hacen uso de bases de datos nacionales para poder explorar la eficacia escolar y han encontrado resultados similares a los de estudios comparativos regionales (Schmelkes et al., 1997; Piñeros y Rodríguez, 1998; Cervini, 2001; Benavides, 2002; Cueto, 2004; Cueto et al., 2006).

\subsection{Eficacia escolar en el Perú}

En el Perú, son pocos los estudios que han abordado el análisis de los procesos escolares al interior de las aulas de clase y no hay estudio alguno que haya explorado el efecto de las mismas en las brechas de rendimiento de los estudiantes, en especial, la socioeconómica. La mayoría de investigaciones en el Perú son de corte transversal, con datos del rendimiento de los alumnos en un solo punto en el tiempo (Banco Mundial, 2001; UMC \& Grade, 2001; Caro, 2004; Asmad et al., 2004; Valdivia, 2004; Agüero \& Cueto, 2004; Cueto et al., 2004). Principalmente, estos estudios han utilizado variables relacionadas con los recursos de la escuela, así como con las características del docente y de las aulas de clases. Cueto et al. (2004), sirviéndose de las bases de datos de la evaluación nacional y analizando los cuadernos de trabajo de los alumnos, hallaron que aquellos docentes que desarrollaban ejercicios de mayor nivel de demanda cognoscitiva y que brindaban adecuada retroalimentación a sus alumnos lograban que estos alcancen mejores resultados.

Además, Cueto et al. $(2004,2006)$ y Cueto et al. (2014) realizaron estudios en los cuales utilizaron medidas a inicios y fin de año escolar para poder identificar las variables asociadas con el aprendizaje de los estudiantes. En el primer estudio, realizado en el año 2003, encontraron que, en aquellas aulas en las que las relaciones entre los alumnos eran cordiales, el rendimiento era mayor. En los estudios realizados en 2003, 2004 y 2006, nuevamente se analizan los cuadernos de trabajo de los alumnos en Matemática y Comunicación, a partir de lo cual se encontró, coincidentemente, que los docentes que desarrollaban ejercicios de mayor demanda cognoscitiva y que brindaban retroalimentación a sus alumnos obtenían mejores resultados en el rendimiento en esas áreas.

En resumen, tomando en cuenta la literatura internacional, latinoamericana y local, son las variables pedagógicas y los procesos escolares los que

4. Se seleccionaron tres grupos de escuelas al interior de cada país: escuelas eficaces, escuelas de eficacia media y escuelas ineficaces. 
tienen un efecto importante sobre el rendimiento de los estudiantes, especialmente aquellas relacionadas con los procesos que se desarrollan al interior del aula de clase. Sin embargo, no existen estudios a nivel local que exploren el efecto de las variables de procesos escolares en las brechas de rendimiento de los estudiantes, en especial, debido a diferencias socioeconómicas.

\section{Preguntas de investigación, objetivos e hipótesis}

El presente estudio tuvo dos objetivos centrales. Cada uno de ellos incluyó preguntas de investigación que fueron respondidas mediante modelos estadísticos descritos en detalle en la sección correspondiente al diseño metodológico. El primer objetivo buscó explorar el efecto que tienen las distintas variables de procesos escolares sobre el rendimiento de los estudiantes en Perú controlando por variables individuales, familiares, contextuales e insumos: $\dot{i}$ Cuál es el tamaño del «efecto escuela»? ¿Cuál es el peso de las variables de procesos escolares en su conjunto? ¿Qué variables de procesos escolares son las más relevantes para explicar el rendimiento de los estudiantes?

Las hipótesis del estudio sobre los efectos de las variables de procesos para estas preguntas se basaron en la literatura existente en el Perú sobre efectividad escolar. Esperábamos que prácticas docentes que han sido identificadas cuantitativamente y cualitativamente como típicas de un "docente efectivo", tales como, planificación anticipada y organización de la clase, cobertura del currículo y retroalimentación a los estudiantes, resulten también significativas en los modelos estadísticos. Asimismo, esperábamos que variables como el liderazgo o gestión técnico-pedagógica del director tuvieran un efecto positivo y significativo sobre el rendimiento.

El segundo objetivo del estudio buscó explorar la asociación entre las variables de procesos escolares y las desigualdades socioeconómicas en el rendimiento de los estudiantes peruanos: ¿Cuál es el efecto que las variables de procesos escolares en la brecha socioeconómica en rendimiento? ¿Cuánto de la brecha se debe a diferencias en las prácticas de los profesores y de los directores?

En el caso de las preguntas para este segundo objetivo, se encontró literatura menos desarrollada y, por tanto, las hipótesis de trabajo fueron más exploratorias. Estas proponían que las variables de procesos escolares tendrían un efecto positivo en reducir las inequidades existentes por niveles socioeconómicos en el rendimiento de los estudiantes. Es decir, los procesos impactarían más a estudiantes de niveles sociales más bajos que a alumnos de niveles más altos. De esta forma, si bien es esperable que las variables de procesos escolares tengan un efecto positivo y significativo a lo largo de todo el sistema educativo, puede ser, también, que la magnitud de los efectos de procesos sea mayor en contextos de mayor vulnerabilidad social (escuelas con menores recursos). 


\section{Metodología}

\subsection{Bases de datos}

El Programa Internacional para la Evaluación de Estudiantes (Pisa) es una evaluación internacional orientada a estudiantes de quince años matriculados en la educación formal secundaria. Las pruebas Pisa están organizadas por la OCDE (Organización para la Cooperación y Desarrollo Económico ${ }^{5}$ ); los participantes son principalmente países industrializados miembros de la OCDE, junto con algunos países en desarrollo que voluntariamente han decidido participar en la evaluación. Las pruebas Pisa se vienen realizando cada 3 años desde el año 2000 y evalúan 3 áreas del conocimiento en los estudiantes: Comprensión Lectora, Matemática y Ciencias. Perú ha participado en 3 rondas de Pisa: 2000, 2009 y 2012. En el presente estudio, se utilizará la última ronda de Pisa disponible para el Perú (2012), que tuvo como énfasis la enseñanza de las matemáticas.

Las bases de datos de Pisa, para el caso del Perú, tienen inferencia a nivel nacional, por área de residencia de la institución educativa y tipo de gestión de la institución educativa. El número de estudiantes evaluados en la última evaluación de Pisa (2012) fue sido de 6035 estudiantes en 240 instituciones educativas. Asimismo, dado el énfasis del presente estudio en aportar al planteamiento de políticas educativas públicas a partir de la identificación de variables escolares que influyan en el rendimiento de los estudiantes a nivel secundario, se precisa estudiar estos aspectos en estudiantes e instituciones educativas de gestión pública. Esta decisión también radica en el enfoque exploratorio de nuestro segundo objetivo, motivo por el cual se decidió prestar atención a un solo tipo de gestión educativa. La siguiente tabla da un alcance de la muestra para el presente estudio.

Tabla 1. Muestra de estudiantes e instituciones educativas

\begin{tabular}{lcccc}
\hline & & Urbano & Rural & Total \\
\hline Estudiantes & $\%$ & 79,3 & 20,7 & 100,0 \\
& $\mathrm{n}$ & $(3771)$ & $(985)$ & $(4756)$ \\
\hline Instituciones educativas & $\%$ & 68,9 & 31,1 & 100,0 \\
& $\mathrm{n}$ & $(126)$ & $(57)$ & $(183)$ \\
\hline
\end{tabular}

Fuente: Elaboración propia

5. Puede consultarse Organisation for Economic Co-operation and Development (s.f.). 


\subsection{Variables}

A continuación, se desarrolla cuál fue la variable dependiente para el presente estudio:

- Rendimiento en Matemática: Se cuenta con cinco puntajes o valores posibles (pvlmath - pv5math) en la prueba de Matemática para los estudiantes. Los puntajes de los alumnos fueron estimados usando Mixed-coefficient Multinomial Logit Model (OECD, 2014); este tipo de modelo estima la distribución de los puntajes de los individuos considerando tanto la información de la prueba (dificultad del ítem) como las características de la población (ej. género, ocupación del padre). Por ello, este modelo nos permitió calcular los diferentes valores posibles en los puntajes del estudiante y relacionarlos con las características del mismo.

A nivel del estudiante y sus familias, se usaron las siguientes variables:

- Índice socioeconómico y cultural de las familias (ESCS): Este refleja las características socioeconómicas y culturales de las familias de los estudiantes que son parte de la evaluación Pisa. Está compuesto por tres indicadores: nivel ocupacional de los padres, nivel educativo de los padres y el índice de posesiones en el hogar; los tres fueron combinados mediante el uso del análisis de componentes principales con la finalidad de maximizar la varianza explicada por cada variable (OECD, 2014).

- Género: Se trata de una variable dicotómica que toma el valor de 1 si el estudiante es mujer y 0 si es hombre.

- Lengua: Esta variable dicotómica toma el valor de 1 si el estudiante habla otra lengua que no sea el castellano en casa y 0 en cualquier otro caso.

- Estructura familiar: Contempla variables dicotómicas relacionadas con la estructura familiar, específicamente vinculadas con la presencia de los padres en los hogares nucleares. Se incluyen variables que toman el valor de 1 si el hogar tiene ambos padres y 0 en cualquier otro caso, y otra variable que toma el valor de 1 cuando en el hogar ninguno de los padres está presente y 0 en cualquier otro caso.

- Edad para el grado: Esta variable dicotómica toma el valor de 1 si el estudiante se encuentra en el grado para su edad y 0 en cualquier otro caso.

- Educación preescolar: Esta variable dicotómica toma el valor de 1 si el estudiante asistió por más de un año a educación preescolar y 0 en cualquier otro caso.

A nivel de la institución educativa, se usan las siguientes variables contextuales:

- IE urbana: Esta variable dicotómica toma el valor de 1 si la escuela se ubica en un área urbana y 0 en cualquier otro caso.

- Proporción de estudiantes mujeres: Esta variable refleja la proporción de estudiantes encuestadas que son mujeres.

- Nivel socioeconómico promedio de la institución educativa: Esta variable indica el nivel socioeconómico promedio de las familias de los alumnos matriculados en la institución educativa. 
- Recursos educativos en la institución ${ }^{6}$ : Este índice refleja si la institución educativa cuenta o no con diferentes recursos educativo para la enseñanza, tales como i) equipos para los laboratorios, ii) materiales educativos, iii) computadoras para la enseñanza, iv) conexión a Internet, v) programas de computación y vi) materiales de consulta (Alpha de Cronbach ${ }^{7}: 0,89$ ).

A nivel del aula, las variables relacionadas prácticas pedagógicas son las siguientes:

- Apoyo de los docentes a los estudiantes: Este índice refleja la percepción de los estudiantes con respecto al apoyo que les brinda el docente de Matemáticas en el aula de clase. Así, se preguntó a los estudiantes con qué frecuencia este realiza lo siguiente: i) muestra interés en el aprendizaje de cada uno de sus estudiantes, ii) brinda ayuda adicional en caso algún estudiante lo necesite, iii) ayuda con el aprendizaje de sus estudiantes, iv) continúa enseñando hasta que sus estudiantes aprendan, v) da la oportunidad a los estudiantes de expresar sus opiniones (Alpha de Cronbach: 0,73).

- Activación cognitiva en las clases de Matemáticas: Este índice refleja la percepción de los estudiantes con respecto a si el docente realiza acciones para estimular cognitivamente a los estudiantes. Se preguntó a los estudiantes qué tan frecuentemente su docente de Matemática realizaba lo siguiente: i) plantea preguntas que generan a los estudiantes reflexionen sobre el problema, ii) desarrolla ejercicios que requieren del estudiante pensar por un largo rato en la solución, iii) indica que los alumnos deben de decidir por cuenta propia los procedimientos a seguir para resolver problemas complejos, iv) formula ejercicios que no requieren una solución obvia o inmediata, v) desarrolla ejercicios en diferentes contextos para saber si se entendieron los conceptos, vi) les enseña a aprender de sus errores, vii) pide que los estudiantes expliquen cómo desarrollan los ejercicios, viii) plantea ejercicios que requieren usar conceptos aprendidos pero en contextos diferentes, ix) propone ejercicios que pueden ser resueltos de diferentes maneras (Alpha de Cronbach: 0,76).

- Clima disciplinario adecuado en la clase de Matemática: Este índice refleja la percepción de los estudiantes con respecto a la disciplina que hay en sus clases de Matemática. Se les preguntó con qué frecuencia sucedía en clase lo siguiente: i) no escuchan lo que dice el docente, ii) hay desorden y ruido durante la clase, iii) los docentes tienen que esperar largo rato para empezar la clase, iv) no pueden trabajar bien en clase, v) no se puede empezar a trabajar sino hasta un buen rato después de empezada la clase (Alpha de Cronbach: 0,78).

6. Los índices fueron construidos usando un análisis factorial confirmatorio (Kline, 2005). Así mismo, en el caso de las escalas relacionadas a los docentes, se volvió binarias las respuestas de los estudiantes.

7. Alpha de Croncach es un coeficiente calculado para medir la fiabilidad de las escalas de medidas. De esta forma, mientras el coeficiente se acerque más a su valor máximo 1 , mayor será la fiabilidad de la escala. 
A nivel de la institución educativa, las variables relacionadas con los procesos educativos son las siguientes:

- Sentido de pertenencia de los estudiantes: Este índice refleja el sentido de pertenencia que tienen los estudiantes con su escuela. Se preguntó a los alumnos qué tan de acuerdo estaban con los siguientes enunciados: i) «siento que no pertenezco a esta escuela», ii) «hago amigos fácilmente en la escuela», iii) «siento que pertenezco a esta escuela», iv) «me siento raro o fuera de sitio en la escuela», v) «los otros estudiantes se parecen a mí», vi) «me siento solo en la escuela», vii) «me siento feliz en la escuela», viii) «las cosas son buenas en mi escuela» y ix) «estoy satisfecho en esta escuela» (Alpha de Cronbach: 0,78).

- Buena relación entre los estudiantes y el docente: Este índice refleja la percepción de los estudiantes sobre la relación que tienen con los docentes de su escuela. Se les preguntó qué tan de acuerdo estaban con los siguientes enunciados: i) los estudiantes se llevan bien con sus docentes, ii) la mayoría de los docentes están interesados en el bienestar de los estudiantes, iii) la mayoría de los docentes realmente escuchan lo que los alumnos tienen que decir, iv) los docentes ayudan a los estudiantes en caso necesiten ayuda extra y v) la mayoría de los docentes tratan a los alumnos de manera justa (Alpha de Cronbach: 0,66).

- Espacios de participación para los docentes en la IE generados por el director: Este índice mide la labor del director para facilitar espacios de diálogo y participación de sus docentes. Se preguntó al director con qué frecuencia realiza las siguientes acciones: i) da oportunidades a los docentes para que formen parte de la toma de decisiones en la institución educativa, ii) compromete a los docentes a que colaboren para construir una cultura de paz escolar para lograr un desarrollo continuo de la institución, iii) hace participar a los docentes en la revisión de las políticas de manejo de recursos al interior de la institución educativa (Alpha de Cronbach: 0,76).

- Autonomía de la IE: Este índice refleja el nivel de autonomía de la escuela en diferentes decisiones relacionadas con el manejo de la escuela. Se preguntó si los docentes y directores asumían la responsabilidad para realizar las siguientes acciones: i) seleccionar docentes para ser contratados, ii) despedir docentes, iii) establecer los salarios de entrada de los docentes, iv) establece los aumentos salariales, v) formular el presupuesto de la escuela, vi) decidir sobre el presupuesto de la escuela, vii) establecer las medidas disciplinarias a los estudiantes, viii) establecer las políticas de evaluación, ix) aprobar qué estudiantes son aceptados en la escuela, $\mathrm{x}$ ) definir los textos escolares que se van usar, xi) determinar el contenido de los cursos, y xii) definir los cursos que se van a dictar (Alpha de Cronbach: 0,71).

La siguiente tabla nos muestra los estadísticos descriptivos de las variables individuales, familiares y escolares consideradas para el presente estudio. 
Tabla 2. Estadísticos descriptivos de las variables individuales, familiares y escolares ${ }^{1 /}$

\begin{tabular}{|c|c|c|c|c|}
\hline & Media & E.E. ${ }^{2 /}$ & Mínimo & Máximo \\
\hline \multicolumn{5}{|l|}{ Variable de estudio } \\
\hline Rendimiento promedio en Matemática & 349,63 & $(3,322)$ & 108,90 & 641,23 \\
\hline \multicolumn{5}{|l|}{ Variables individuales y familiares } \\
\hline Edad en meses & 189,39 & $(0,049)$ & 183,96 & 195,00 \\
\hline Mujeres (\%) & 51,37 & $(2,011)$ & 0 & 1 \\
\hline En edad para el grado (\%) & 67,47 & $(1,423)$ & 0 & 1 \\
\hline \multicolumn{5}{|l|}{ Educación temprana (\%) } \\
\hline No asistió & 16,59 & $(0,988)$ & 0 & 1 \\
\hline Un año o menos & 24,95 & $(0,892)$ & 0 & 1 \\
\hline Más de dos años & 55,34 & $(1,450)$ & 0 & 1 \\
\hline No respondió & 3,12 & $(0,313)$ & 0 & 1 \\
\hline Índice socioeconómico y cultural (ISCS) & $-0,24$ & $(0,036)$ & $-3,41$ & 2,53 \\
\hline \multicolumn{5}{|l|}{ Estructura familiar (\%) } \\
\hline Monoparental & 13,79 & $(0,525)$ & 0 & 1 \\
\hline Nuclear & 70,07 & $(0,802)$ & 0 & 1 \\
\hline Extendido y otros & 3,28 & $(0,325)$ & 0 & 1 \\
\hline No respondió & 12,86 & $(0,654)$ & 0 & 1 \\
\hline \multicolumn{5}{|l|}{ Lengua en el hogar (\%) } \\
\hline Castellano & 87,78 & $(1,298)$ & 0 & 1 \\
\hline Otra lengua & 8,03 & $(1,204)$ & 0 & 1 \\
\hline No respondió & 4,20 & $(0,351)$ & 0 & 1 \\
\hline \multicolumn{5}{|l|}{ Variables contextuales } \\
\hline IE ubicada en un área urbana (\%) & 76,30 & $(2,888)$ & 0 & 1 \\
\hline Nivel socioeconómico promedio de la escuela & $-0,24$ & $(0,036)$ & $-1,88$ & 0,90 \\
\hline Proporción de estudiantes mujeres (\%) & 49,80 & $(1,958)$ & 0 & 1 \\
\hline Recursos educativos en la institución educativa & $-0,13$ & $(0,044)$ & $-0,94$ & 1,56 \\
\hline \multicolumn{5}{|l|}{ Prácticas pedagógicas de los docentes } \\
\hline Clima de disciplina adecuado en el aula & 0,01 & $(0,049)$ & $-2,55$ & 1,50 \\
\hline Activación cognitiva en las clases de Matemáticas & $-0,03$ & $(0,032)$ & $-2,91$ & 0,95 \\
\hline Apoyo de los docentes a los estudiantes en clase & 0,00 & $(0,035)$ & $-2,58$ & 1,35 \\
\hline \multicolumn{5}{|l|}{ Procesos escolares en la IE } \\
\hline Relaciones entre estudiantes y docentes & 0,01 & $(0,030)$ & $-1,82$ & 0,89 \\
\hline $\begin{array}{l}\text { Espacios de participación en la IE generados } \\
\text { por el director }\end{array}$ & 0,00 & $(0,085)$ & $-1,82$ & 1,99 \\
\hline Autonomía escolar & $-0,96$ & $(0,156)$ & $-5,03$ & 2,86 \\
\hline Sentido de pertenencia de los estudiantes & 0,06 & $(0,026)$ & $-1,33$ & 1,23 \\
\hline
\end{tabular}

1/ Se incluyeron variables dicotómicas para aquellas variables que tenían valores perdidos con la finalidad de controlar por el posible sesgo que pueda originar en la muestra para los análisis. 2/ El error estándar es calculado usando el diseño muestral.

$\mathrm{N}=4213$

Fuente: Elaboración propia 


\subsection{Análisis estadístico}

Para poder responder las preguntas de investigación planteadas en el presente estudio, se realizó un análisis de regresión lineal jerárquica (Raudenbush \& Bryck, 2002; Goldstein, 2003). La decisión de usar este tipo de herramienta estadística responde a los siguientes aspectos: i) dado el carácter jerárquico de las bases de datos, el realizar una regresión lineal en la que se asume independencia de las observaciones llevaría a subestimar los errores estándar, lo cual puede hacer que consideremos efectos como significativos cuando no lo son; ii) el análisis de regresión multinivel nos permite separar la varianza de la variable dependiente de acuerdo con cada uno de los niveles que se considere; y iii) nos permite estimar las interacciones entre los diferentes niveles considerados en el estudio (escuela y aula). Por ello, se va a usar esta herramienta estadística para modelar el rendimiento de los estudiantes.

Se considerarán dos niveles para el presente estudio: i) el nivel del estudiante y su familia, y ii) el nivel de la escuela. Ello implica descomponer la variabilidad del rendimiento en cada uno de estos niveles. A continuación, se detalla el modelo:

Nivel del estudiante y su familia

$$
Y_{i j}=\beta_{0 j}+\beta_{1 j} E_{i j}+\beta_{2 j} N E_{i j}+\beta_{3 j} F_{i j}+\varepsilon_{i j}
$$

Nivel de la escuela

$$
\begin{aligned}
& \beta_{0 j}=\gamma_{o o}+\gamma_{o 1} \mathrm{CE}+\gamma_{\mathrm{o} 2} \mathrm{IE}+\gamma_{\mathrm{o} 3} \mathrm{PD}+\gamma_{04} \mathrm{PE}+\mu_{\mathrm{oj}} \\
& \beta_{1 j}=\gamma_{10} \\
& \beta_{2 j}=\gamma_{20}+\gamma_{21} \mathrm{CE}+\gamma_{22} \mathrm{IE}+\gamma_{23} \mathrm{PD}+\gamma_{24} \mathrm{PE}+\mu_{2 \mathrm{j}} \\
& \beta_{3 j}=\gamma_{30}
\end{aligned}
$$

\section{Supuestos del modelo}

i) $\mu_{\mathrm{oj}} \sim \mathrm{N}\left(0, \lambda^{2}\right)$, i) $\mu_{2 \mathrm{j}} \sim \mathrm{N}\left(0, \Omega^{2}\right)$, iii) $\varepsilon_{\mathrm{ij}} \sim \mathrm{N}\left(0, \Phi^{2}\right)$, y iv $) \operatorname{cov}\left(\mu_{\mathrm{oj}}, \varepsilon_{\mathrm{ij}}\right)=0$, $\operatorname{cov}\left(\mu_{\mathrm{oj}}, \mu_{2 \mathrm{j}}\right)=0, \operatorname{cov}\left(\mu_{2 \mathrm{j}}, \varepsilon_{\mathrm{ij}}\right)=0$

\section{Variables}

$\mathrm{Y}=$ Rendimiento de los estudiantes, $\mathrm{E}=$ Características de los estudiantes, NSE=Nivel socioeconómico de las familias, $\mathrm{F}=$ Características de las familias, $\mathrm{CE}=$ Contexto escolar, $\mathrm{IE}=$ Insumos escolares, $\mathrm{PD}=$ Prácticas docentes y $\mathrm{PE}=$ Procesos escolares en la IE 
De esta manera, reemplazando de las ecuaciones 2 al 5 en 1 , se obtiene la ecuación 6.

$$
\begin{aligned}
\mathrm{Y}_{\mathrm{ijk}}= & \gamma_{\mathrm{oo}}+\gamma_{\mathrm{o} 1} \mathrm{CE}+\gamma_{\mathrm{o} 2} \mathrm{IE}+\gamma_{\mathrm{o} 3} \mathrm{PD}+\gamma_{\mathrm{o} 4} \mathrm{PE}+\gamma_{1 \mathrm{o}} \mathrm{E}_{\mathrm{ij}} \\
& +\gamma_{20}{ }^{*} \mathrm{NSE}+\gamma_{21} \mathrm{CE}^{\star} \mathrm{NSE}+\gamma_{22} \mathrm{IE}^{\star} \mathrm{NSE}+\gamma_{23} \mathrm{PD}^{\star} \mathrm{NSE} \\
& +\gamma_{24} \mathrm{PE}^{\star} \mathrm{NSE}+\mu_{2 \mathrm{j}}{ }^{\star} \mathrm{NSE}+\gamma_{30} \mathrm{~F}_{\mathrm{ij}}+\mu_{\mathrm{oj}}+\varepsilon_{\mathrm{ij} \mathrm{k}}
\end{aligned}
$$

Este método de modelación, marca una diferencia con los métodos que se han estado empleando, dado que, a diferencia de otros estudios, se realiza una modelación de las brechas de rendimiento socioeconómicas de los estudiantes. Ello genera que la variable dependiente ya no sea solo el rendimiento en la prueba, sino el coeficiente de las diferencias socioeconómicas a nivel del estudiante (ecuación 4).

Así, para responder a las preguntas correspondientes al primer objetivo de investigación relacionado con la magnitud del efecto de las variables de procesos escolares sobre el rendimiento de los estudiantes, se utiliza la ecuación 6 . En ella, los vectores $\gamma_{\mathbf{o} 2}, \gamma_{\mathbf{o} 3} \mathrm{y} \gamma_{\mathbf{o 4}}$ contienen los efectos de las variables de insumos escolares, prácticas docentes y procesos escolares en la institución educativa en el rendimiento promedio de los estudiantes.

Para responder a las preguntas correspondientes al segundo objetivo de investigación relacionado con el efecto de las variables de procesos escolares en las brechas socioeconómicas de los estudiantes, también se emplea la ecuación 6. En esta, los vectores $\gamma_{22}, \gamma_{23}$ y $\gamma_{24}$ contiene los efectos de las variables de insumos, prácticas docentes y procesos escolares sobre las brechas socioeconómicas de los estudiantes en las instituciones educativas públicas.

\section{Resultados}

\subsection{La relación entre el nivel socioeconómico de la familia y el rendimiento en matemática}

Un primer aspecto es determinar la magnitud y sentido de la asociación entre el nivel socioeconómico de las familias y el rendimiento de los estudiantes. La siguiente figura muestra que existe una relación positiva y significativa entre el rendimiento de los estudiantes y su nivel socioeconómico. 
Figura 2. Rendimiento promedio de los estudiantes en Matemática de acuerdo con el nivel socioeconómico de las familias

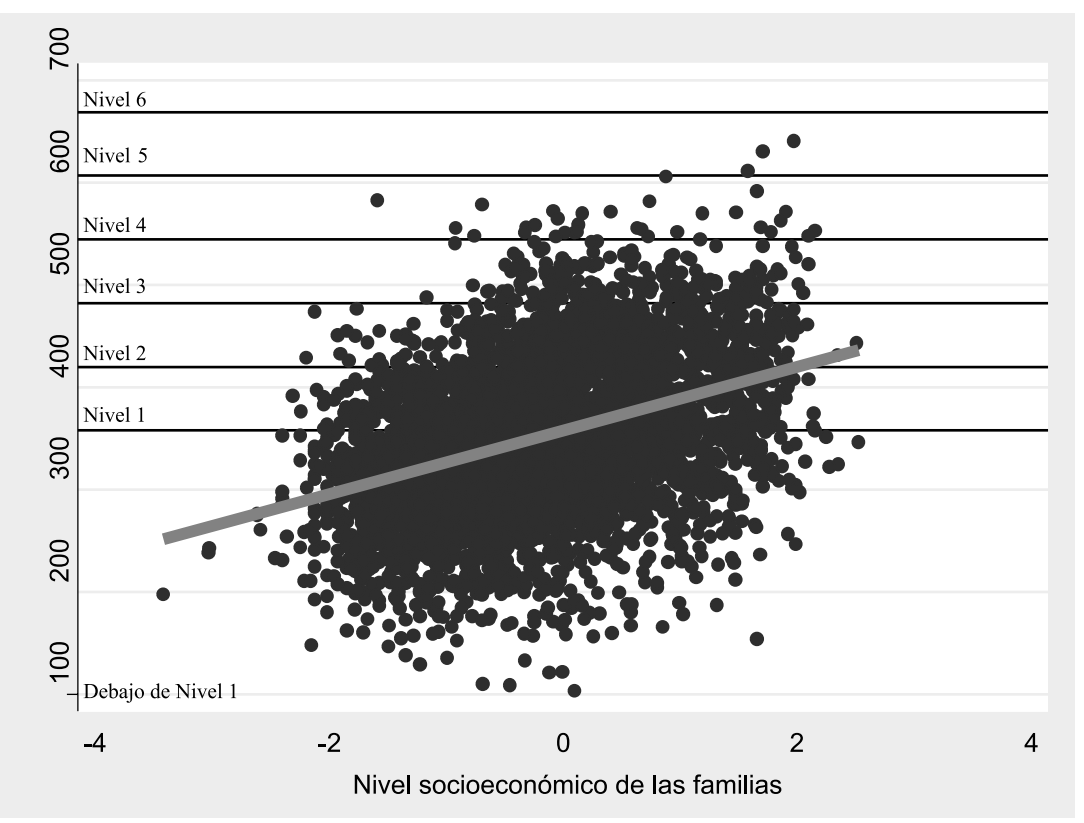

Fuente: OECD (2013)

Elaboración propia

Sin embargo, ¿cuál es la magnitud de la brecha entre estudiantes de alto y bajo nivel socioeconómico en las instituciones educativas públicas? La tabla 3 muestra los puntajes promedios en Matemática por quintiles del nivel socioeconómico de los estudiantes. Tal como se puede observar, la diferencia en los puntajes entre estudiantes del quintil más alto y el quintil más bajo es de 80 puntos, una diferencia que es estadísticamente significativa. Así mismo, se aprecia que la diferencia en el rendimiento entre el primer quintil y cada uno de los otros quintiles es estadísticamente significativa. 
Tabla 3. Puntajes promedio por quintiles de nivel socioeconómico de las familias $(\mathrm{N}=4312)$

\begin{tabular}{lccc}
\hline & \multicolumn{2}{c}{ Puntaje en Matemática } & \multirow{2}{*}{ Diferencia con el primer quintil } \\
\cline { 2 - 3 } & Promedio & Error estándar & \\
\hline Primer (menor NSE) & 309,4 & $(3,0)$ & - \\
Segundo & 329,8 & $(2,9)$ & $20,4^{\star}$ \\
Tercero & 355,7 & $(3,4)$ & $46,3^{\star}$ \\
Cuarto & 369,4 & $(3,9)$ & $60,1^{\star}$ \\
Quinto (mayor NSE) & 389,7 & $(4,7)$ & $80,3^{\star}$ \\
\hline
\end{tabular}

* Diferencias estadísticamente significativas al 5\% de acuerdo con el t-test para muestras independientes

Fuente: OECD (2013)

Elaboración propia

En cuanto al porcentaje de estudiantes por niveles de desempeño y niveles socioeconómicos, la siguiente tabla muestra que el porcentaje de estudiantes que no domina o domina de manera muy básica las habilidades matemáticas para su edad es del $78 \%$ en el quintil más bajo; y de 64\%, en el quintil más alto. De esta manera, se puede observar que son los estudiantes de quintiles de menores niveles socioeconómicos los que peores resultados educativos obtienen. Sin embargo, también, alarma que incluso aquellos estudiantes con mejores niveles socioeconómicos obtienen resultados bastantes pobres en el sistema educativo público.

Tabla 4. Porcentaje de estudiantes por niveles de desempeño y nivel socioeconómico en Matemáticas $(\mathrm{N}=4312)$

\begin{tabular}{lccccc}
\hline & \multicolumn{5}{c}{ Quintiles socioeconómicos } \\
\cline { 2 - 6 } & Q1 & Q2 & Q3 & Q4 & Q5 \\
\hline Nivel 6 (encima de 669,3) & - & - & - & - & - \\
Nivel 5 (entre 607,0 y 669,3) & 0,0 & 0,8 & 1,8 & 0,0 & 0,0 \\
Nivel 4 (entre 544,7 y 607,0) & 5,1 & 8,1 & 0,0 & 0,0 & 0,1 \\
Nivel 3 (entre 482,4 y 544,7) & 24,7 & 0,0 & 0,0 & 0,2 & 1,2 \\
Nivel 2 (entre 420,1 y 482,4) & 0,0 & 0,0 & 3,0 & 7,3 & 13,1 \\
Nivel 1 (entre 357,8 y 420,1) & 0,0 & 18,5 & 23,2 & 31,8 & 31,9 \\
Debajo de nivel 1 (debajo de 357,8) & 78,3 & 68,2 & 50,9 & 43,6 & 32,3 \\
\hline
\end{tabular}

Fuente: OECD (2013)

Elaboración prop ia

Finalmente, en la figura 3, se puede apreciar cuánto de la varianza entre individuos y entre escuelas explica el nivel socioeconómico de las familias. Se puede apreciar que, a nivel individual, el nivel socioeconómico solo explica el 
$2 \%$ de la variabilidad entre estudiantes, mientras que, a nivel de instituciones educativas, el nivel socioeconómico explica el $40 \%$ de la variabilidad del rendimiento en Matemática. Estos resultados evidencian que la diferencia de los puntajes de los estudiantes al interior de las instituciones educativas no depende tanto del nivel socioeconómico de los estudiantes, sino que son otros factores los que estarían jugando un rol importante para determinar los puntajes de los estudiantes en Matemática. En cambio, el nivel socioeconómico de las familias sí estaría jugando un rol importante para explicar las diferencias en el rendimiento en Matemática entre instituciones educativas, aspecto que estaría mostrando el nivel de segregación socioeconómica en las instituciones educativas públicas.

Figura 3. Varianza explicada por el nivel socioeconómico de las familias entre estudiantes y entre escuelas

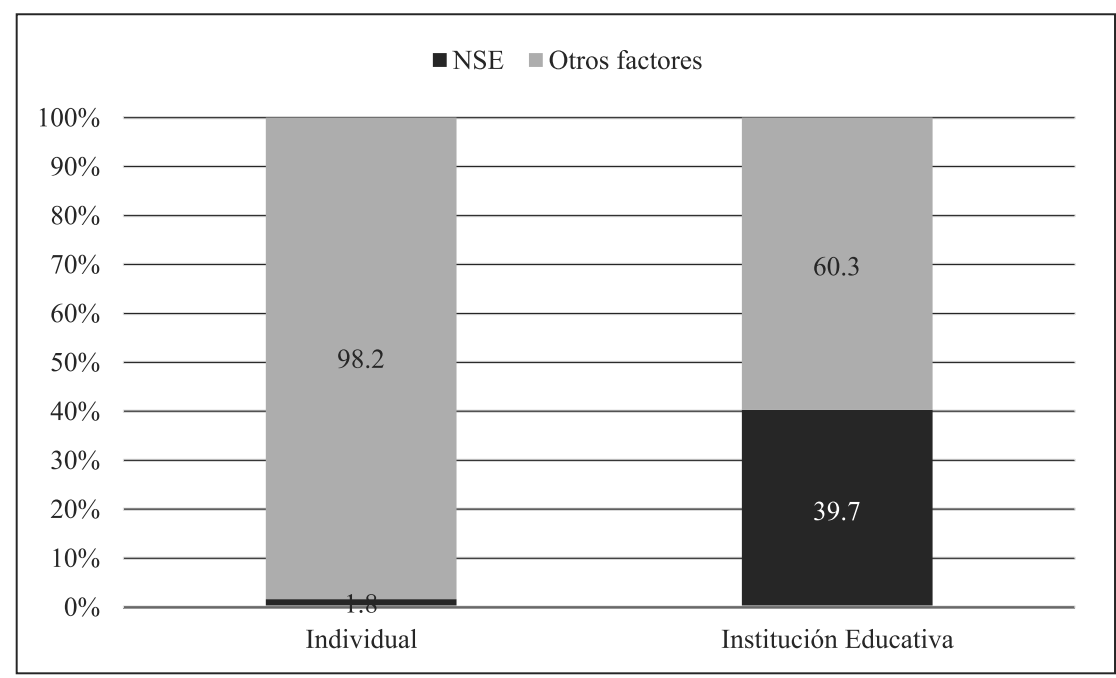

Estudiantes: 4312

Instituciones educativas: 167

Fuente: OECD (2013)

Elaboración propia

\subsection{El efecto de las variables de procesos escolares en el rendimiento} de los estudiantes

Como se mencionó anteriormente, el primer objetivo de este estudio es explorar la asociación entre las variables escolares y el rendimiento de los estudiantes, con especial énfasis en las variables de procesos educativos en el aula y la institución educativa. La tabla 5 muestra que existe una correlación positiva y significativa entre el rendimiento promedio en Matemática y las variables contextuales, prácticas pedagógicas y procesos escolares en la institución educativa. Así, estudiantes

168 I de instituciones educativas urbanas, de mayor nivel socioeconómico promedio en la institución educativa, con mayor proporción de estudiantes en edad para el 
grado, de instituciones educativas con mejor calidad (recursos, infraestructura y tamaño) y con mayor proporción de estudiantes con educación preescolar, son aquellas que obtienen mejores resultados en Matemática.

En cuanto a la relación entre el rendimiento promedio en Matemática y las variables de procesos escolares en el aula y la institución educativa, se ha encontrado que aquellas instituciones educativas donde los docentes mantienen un clima de disciplina adecuado y los estudiantes en promedio tienen mayor sentido de pertenencia con la institución son aquellas en las que los estudiantes obtienen mejores resultados en Matemáticas.

Tabla 5. Correlación entre las variables escolares y el rendimiento promedio en Matemática

\begin{tabular}{|c|c|c|}
\hline & $\mathrm{r}$ & \\
\hline \multicolumn{3}{|l|}{ Variables contextuales } \\
\hline IE ubicada en un área urbana & 0,28 & * \\
\hline Nivel socioeconómico promedio de la escuela & 0,48 & * \\
\hline Proporción de estudiantes mujeres & 0,06 & \\
\hline Índice de recursos educativos & 0,24 & * \\
\hline \multicolumn{3}{|l|}{ Prácticas pedagógicas de los docentes } \\
\hline Clima de disciplina adecuado en el aula & 0,14 & * \\
\hline Activación cognitiva en las clases de Matemáticas & 0,06 & \\
\hline Apoyo de los docentes a los estudiantes en clase & 0,02 & \\
\hline \multicolumn{3}{|l|}{ Procesos escolares en la IE } \\
\hline Relaciones entre estudiantes y docentes & 0,04 & \\
\hline Espacios de participación en la IE generados por el director & 0,00 & \\
\hline Sentido de pertenencia de los estudiantes & 0,14 & * \\
\hline Autonomía escolar & 0,00 & \\
\hline
\end{tabular}

${ }^{*}$ Correlación estadísticamente significativa al 5\%

Estudiantes: 4312

Instituciones educativas: 167

Fuente: OECD (2013)

Elaboración propia

De esta manera, se ha podido observar que existe asociación entre las variables escolares y el rendimiento de los estudiantes en Matemática. Sin embargo, estas relaciones no cuentan con ningún tipo de control o ajuste estadístico. Por ello, la tabla 6 muestra los resultados de la regresión lineal jerárquica para explicar el rendimiento promedio de los estudiantes en las IE, de forma tal que sea posible tener los efectos netos de cada una de las variables escolares incluidas en los modelos ${ }^{8}$.

8. Cabe señalar que, para la estimación del modelo multinivel, se utilizaron los pesos 
Se puede apreciar que son pocas las variables a nivel de la IE que están asociadas con el rendimiento de los estudiantes en Matemática. En lo que respecta a las variables contextuales, el nivel socioeconómico de la escuela tiene un efecto positivo y significativo $\left(\gamma_{02}=0,18 \mathrm{DE}, \mathrm{p}<0,01\right)$ sobre el rendimiento de los estudiantes e, incluso, es el de mayor magnitud. Además, se aprecia que los estudiantes de IE con mayores recursos educativos $\left(\gamma_{05}=0.09 \mathrm{DE}, \mathrm{p}<0,05\right)$ o que se ubican en zonas urbanas $\left(\gamma_{01}=0.08 \mathrm{DE}, \mathrm{p}<0.10\right)$ tienen un mayor rendimiento en Matemática. En cuanto a las variables relacionadas con las practicas pedagógicas y los procesos escolares, se aprecia que aquellos docentes que mantienen un clima de disciplina adecuado en su aula de clase $\left(\gamma_{06}=0,14 \mathrm{DE}\right.$, $\mathrm{p}<0,01)$ obtienen mejores resultados en Matemáticas. Este aspecto puede estar vinculado con que aquellos docentes que pueden guardar un orden en su aula de clase facilitan el desarrollo de los procesos de enseñanza-aprendizaje.

Tabla 6. Efectos netos de las variables escolares en el rendimiento de los estudiantes en Matemática

\begin{tabular}{lcccc}
\hline & Notación & Efecto & Error & Efecto STD \\
\cline { 2 - 5 } & $\gamma_{00}$ & 319,2 & $(5,61) * * *$ & - \\
\hline Variables contextuales & & & & \\
$\quad$ IE ubicada en un área urbana & $\gamma_{01}$ & 12,7 & $(6,79)+$ & 0,08 \\
$\quad$ Nivel socioeconómico promedio de la escuela & $\gamma_{02}$ & 23,1 & $(7,38) * * *$ & 0,18 \\
$\quad \begin{array}{l}\text { Proporción de estudiantes mujeres } \\
\text { Índice de recursos educativos }\end{array}$ & $\gamma_{03}$ & $-4,7$ & $(13,65)$ & $-0,01$ \\
\hline Prácticas pedagógicas de los docentes & $\gamma_{05}$ & 7,9 & $(3,09) *$ & 0,09 \\
$\quad$ Clima de disciplina adecuado en el aula & $\gamma_{06}$ & 15,2 & $(4,18) * *$ & 0,14 \\
$\quad$ Activación cognitiva en las clases de Matemáticas & $\gamma_{07}$ & $-4,5$ & $(6,91)$ & $-0,03$ \\
$\quad$ Apoyo de los docentes a los estudiantes en clase & $\gamma_{08}$ & 10,0 & $(6,76)$ & 0,08 \\
\hline Procesos escolares en la IE & & & & \\
$\quad$ Relaciones entre estudiantes y docentes & $\gamma_{09}$ & $-7,1$ & $(7,60)$ & $-0,05$ \\
Espacios de participación en la IE generados & $\gamma_{010}$ & 3,5 & $(2,38)$ & 0,05 \\
por el director & $\gamma_{04}$ & $-0,5$ & $(9,76)$ & 0,00 \\
$\quad$ Sentido de pertenencia de los estudiantes & $\gamma_{011}$ & 1,4 & $(1,68)$ & 0,03 \\
$\quad$ Autonomía escolar & & & & \\
\hline
\end{tabular}

${ }^{* * *} \mathrm{p}<0,01 ;{ }^{* *} \mathrm{p}<0,01 ;{ }^{*} \mathrm{p}<0,05 ;+\mathrm{p}<0,10$

Estudiantes: 4312

Instituciones educativas: 167

Nota: Se incluyeron como controles a nivel individual la edad en meses del estudiante, la estructura familiar, la lengua que se habla en casa, el nivel socioeconómico de las familias, el sexo del estudiante, si se encuentra en la edad para el grado y si asistió a educación preescolar. Fuente: Elaboración propia análisis de regresión, se usó el software estadístico HLM 6.0. 
Por otro lado, en relación con el porcentaje de varianza que explican las variables escolares, la siguiente tabla muestra que las variables individuales y familiares explican el 30\% de la variabilidad entre instituciones educativas. Por su parte, las variables contextuales de la IE explican el $21 \%$ de la varianza entre instituciones educativas y las variables de procesos escolares explican el 10\% de la varianza entre instituciones educativas.

Tabla 7. Varianza estimada y porcentaje de varianza explicada entre instituciones educativas en el puntaje promedio de los estudiantes en Matemática

\begin{tabular}{|c|c|c|}
\hline & \multicolumn{2}{|c|}{ Varianza entre escuelas } \\
\hline & Estimado & $\begin{array}{c}\text { \% Varianza } \\
\text { explicada }\end{array}$ \\
\hline Sin controles & $2014^{* * *}$ & - \\
\hline Individuales & $1413^{* * *}$ & 30 \\
\hline Individuales + contextuales & $991^{\star * *}$ & 21 \\
\hline Individuales + contextuales + procesos escolares & $795^{* * *}$ & 10 \\
\hline
\end{tabular}

${ }^{* * *} \mathrm{p}<0,01 ;{ }^{* *} \mathrm{p}<0,01 ;{ }^{*} \mathrm{p}<0,05 ;+\mathrm{p}<0,10$

Estudiantes: 4312

Instituciones Educativas: 167

Fuente: Elaboración propia

\subsection{El efecto de las variables de procesos escolares en las brechas de rendimiento por nivel socioeconómico}

El segundo objetivo del presente estudio es explorar qué variables escolares tienen un efecto en las brechas de rendimiento debido a diferencias socioeconómicas de los estudiantes y sus familias. Así, uno de los primeros aspectos fue evaluar si el componente aleatorio asociado al coeficiente de las diferencias socioeconómicas entre estudiantes varía entre instituciones educativas. Los resultados del análisis indican que existen diferencias estadísticamente significativas entre las brechas de rendimiento debido a diferencias socioeconómicas entre instituciones educativas $(\chi 2=295,6 ; p<0,01)$. De esta forma, para los demás modelos que se estiman, se mantiene este componente aleatorio, de manera que permita controlar las características no observables en las diferencias de las brechas de rendimiento socioeconómicas.

La siguiente tabla muestra los resultados del modelo lineal jerárquico con coeficientes aleatorios. Se puede observar que la brecha de rendimiento debido a diferencias socioeconómicas entre estudiantes es de ocho puntos, una vez que se mantienen constante las características individuales, familiares y escolares de los estudiantes en instituciones educativas públicas. En cuanto al efecto de variables contextuales, las brechas socioeconómicas son mayores en aquellas instituciones educativas con un mayor nivel socioeconómico 
$\left(\gamma_{122}=0,07 \mathrm{DE} ; \mathrm{p}<0,05\right)$. En cuanto a las variables de prácticas pedagógicas y procesos escolares, se encontró que las brechas socioeconómicas son mayores en instituciones educativas donde existe una buena relación entre estudiantes y docentes $\left(\gamma_{128}=0.06 \mathrm{DE}, \mathrm{p}<0.10\right)$, mientras que son menores en las instituciones educativas en las que los estudiantes en promedio tienen un mayor sentido de pertenencia hacia su institución educativa $\left(\gamma_{1210}=-0,05 \mathrm{DE} ; \mathrm{p}<0,10\right)$.

Tabla 8. Efectos de las variables escolares en el rendimiento promedio y las brechas por niveles socioeconómicos en las IE

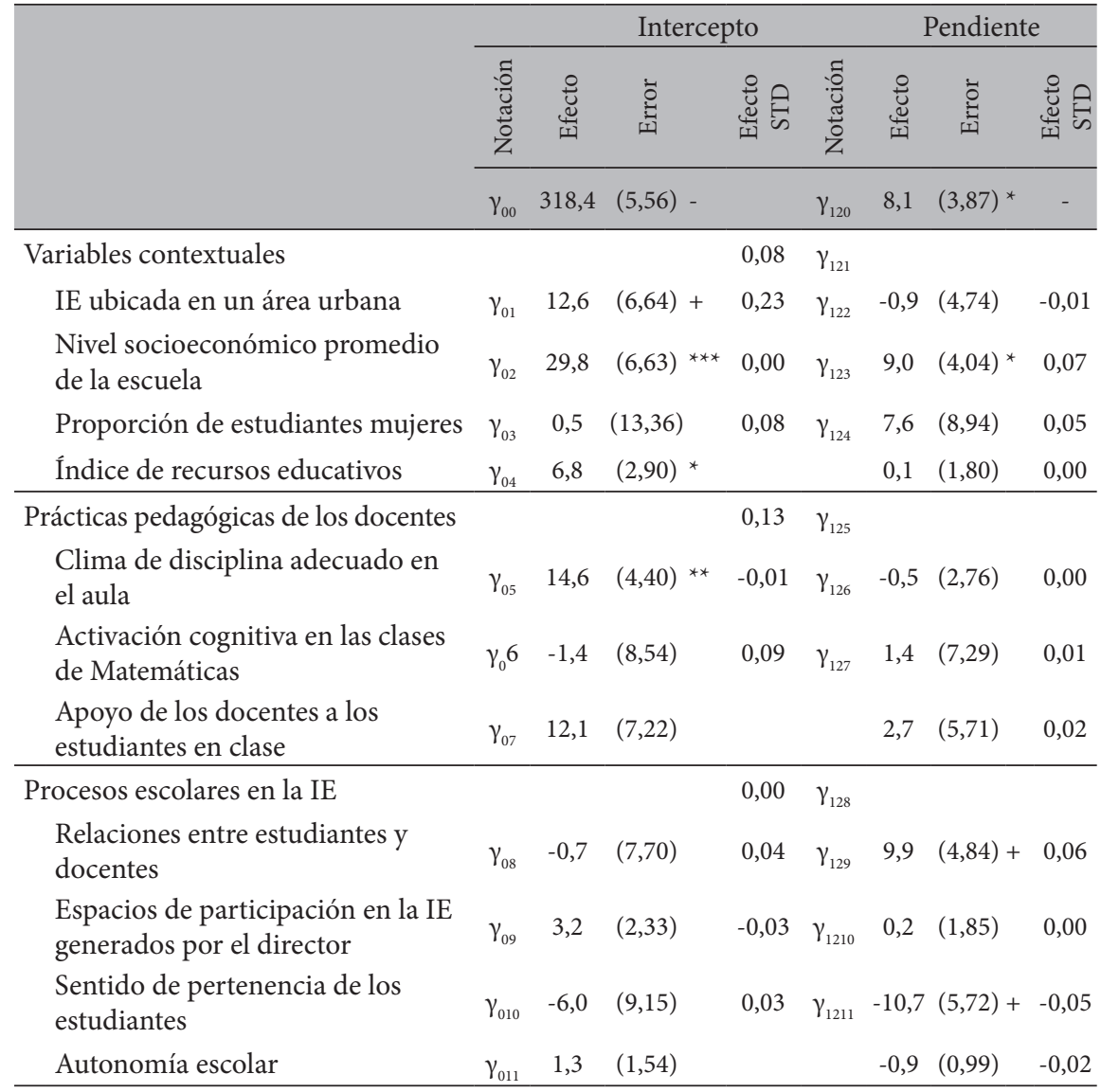

${ }^{* *} \mathrm{p}<0,01 ;{ }^{* *} \mathrm{p}<0,01 ;{ }^{*} \mathrm{p}<0,05 ;+\mathrm{p}<0,10$

Estudiantes: 4312

Instituciones educativas: 167

Nota: Se incluyeron como controles a nivel individual la edad en meses del estudiante, la estructura familiar, la lengua que se habla en casa, el nivel socioeconómico de las familias, el sexo del estudiante, si se encuentra en la edad para el grado y si asistió a educación preescolar. Fuente: Elaboración propia 
En cuanto a la varianza explicada, se puede apreciar, en la tabla 9, que las variables individuales del alumnado y las variables contextuales explican el $4 \%$ de la varianza del rendimiento entre instituciones educativas. Mientras, las variables de procesos escolares explican el $2 \%$ de la varianza cuando son incluidas para explicar las brechas de rendimiento debido a diferencias socioeconómicas.

Tabla 9. Varianza estimada y porcentaje de varianza explicada entre instituciones educativas por variables escolares en las brechas de rendimiento en Matemática

\begin{tabular}{lcc}
\hline & \multicolumn{2}{c}{ Varianza entre escuelas } \\
\cline { 2 - 3 } & Estimado & $\begin{array}{c}\text { \% Varianza } \\
\text { explicada }\end{array}$ \\
\hline Individuales + contextuales & 721 & 4 \\
\hline Individuales + contextuales + procesos escolares (aula e IE) & 686 & 2 \\
\hline
\end{tabular}

${ }^{* *} \mathrm{p}<0,01 ;{ }^{* *} \mathrm{p}<0,01 ;{ }^{\star} \mathrm{p}<0,05 ;+\mathrm{p}<0,10$

Estudiantes: 4312

Instituciones educativas: 167

Fuente: Elaboración propia

\section{Conclusiones}

El presente estudio utilizó las bases de datos de Pisa 2012 con el objetivo de explorar qué variables escolares están asociadas al rendimiento de estudiantes de secundaria y, además, ayudan a explicar las brechas de rendimiento debido a diferencias socioeconómicas de los estudiantes. En función de ello, se partió de los modelos de eficacia escolar para analizar distintas variables de procesos escolares, controlando variables individuales, familiares y contextuales de las IE.

En primer lugar, se encontró que las variables contextuales de la IE tienen un peso importante en la predicción del rendimiento de los estudiantes en Matemáticas. Así, la zona donde se encuentra la IE tiene un efecto positivo y significativo en el rendimiento de los estudiantes. Es decir, estudiantes de IE en zonas urbanas tienen un mayor rendimiento en Matemática que sus pares en zonas rurales. Este resultado alerta, una vez más, sobre la inequidad en la educación peruana según zona de residencia tal como otros estudios desarrollados por la Unidad de Medición de la Calidad Educativa del Ministerio de Educación han señalado $(2004,2006)$, motivo por el cual es pertinente seguir emprendiendo esfuerzos para identificar las variables contextuales y escolares relacionadas con estas diferencias. Asimismo, resulta importante que el gasto público y las estrategias en educación se potencien hacia las zonas rurales.

Por otro lado, los resultados muestran que aquellos estudiantes que asisten a una IE en la que el promedio de familias tiene mayor nivel socioeconómico obtienen mejores resultados educativos que aquellos que estudian en 
una institución en la que el promedio pertenece a menores niveles socioeconómicos. También, se halló que aquellas instituciones educativas que cuentan con diferentes insumos escolares, tales como equipos para los laboratorios, materiales educativos, computadoras, acceso a Internet, softwares educativos y materiales de consulta, son las que obtienen mejores resultados de rendimiento en Matemática. Respecto a ello, el Ministerio de Educación del Perú viene desarrollando diferentes políticas para mejorar los materiales que utilizan los estudiantes; sin embargo, estas son centradas en el nivel primario (soporte pedagógico), motivo por el cual es necesario el desarrollo de programas a nivel secundario que mejoren los sistemas de entrega y dotación de recursos educativos a las IE.

En línea con lo anterior, vamos comprendiendo que variables contextuales vinculadas a menores recursos económicos y materiales de la IE y la familia se relacionan de manera directa con el rendimiento académico más bajo en los estudiantes. Ello coincide con los hallazgos en los estudios de Benavides et al. (2014) y Guadalupe et al. (2013), lo cual, fortalece la idea de que la variable socioeconómica no solo facilita estimar efectos al interior del aula, sino también aquellos relacionados con el contexto escolar. Debido a que se trata de un resultado a nivel contextual, se refuerza la idea de que sus efectos pueden modificarse a partir de políticas públicas. Para ello, sería necesario indagar más acerca de las variables contextuales alteradas por un bajo nivel socioeconómico y que, en consecuencia, alteran el rendimiento escolar. Asimismo, se atribuye la importancia de seguir indagando sobre las variables individuales y contextuales que, según los resultados de este estudio y de la literatura revisada, son elementales para comprender las diferencias en el rendimiento académico (Benavides et al., 2014; Miranda, 2008).

Por otro lado, resultó interesante identificar que los procesos escolares a nivel del aula mostraron importantes efectos sobre el rendimiento en Matemáticas. Así, un clima disciplinario dentro del aula se relaciona con mejores resultados educativos en Matemáticas, a comparación de aquellas aulas donde el docente no mantiene un clima de disciplina. De esta manera, de forma similar a los resultados de Llece $(2001,2010)$, se aprecia que asegurar un clima organizado y disciplinado en el aula, donde el docente mantenga el orden y los estudiantes no lo interrumpan, favorece el aprendizaje de los contenidos de la clase. Debido a ello, debe trabajarse en la formación docente (técnica o universitaria) respecto a estrategias de manejo del clima del aula, puesto que aquellos docentes que utilicen estrategias para mantener el orden en su clase y desarrollar las actividades académicas planificadas podrán promover mejor el rendimiento en sus estudiantes.

En relación con las brechas de rendimiento debido a diferencias socioeconómicas, se encontró que, en las IE que cuentan con un mayor promedio de familias de mayor nivel socioeconómico, las brechas de rendimiento por nivel socioeconómico son mayores. Así, nuevamente, hallamos que la segregación socioeconómica a nivel escolar estaría exacerbando las brechas de rendimiento 
entre los estudiantes del sector público. No obstante, este resultado también alerta que, en escuelas públicas, tanto los estudiantes de un menor nivel socioeconómico como aquellos con mejores niveles de bienestar obtienen resultados bastante pobres dentro del sistema educativo. Ello concuerda con las evaluaciones internacionales en las que los estudiantes del Perú aparecen bastante atrasados y, también, representa un indicador que la agenda política debe priorizar (Llece, 2010; OECD, 2013).

Asimismo, en relación con los procesos escolares, aquellas IE donde el promedio de estudiantes tiene una buena relación con sus docentes de Matemática son en las que las brechas de rendimiento por nivel socioeconómico son mayores. Debido a ello, es necesario que se elaboren estrategias para promover el clima escolar en las IE. Diferentes estudios a nivel nacional han encontrado la importancia del clima escolar en el rendimiento de los estudiantes (Llece, 2001, 2010). Por ello, promover relaciones positivas, en las que los estudiantes se sientan apoyados y escuchados por el docente, posibilitarán un mejor impacto en el rendimiento escolar. Así también, es pertinente seguir indagando sobre las características del clima escolar en las IE peruanas para permitir implementar políticas educativas que intervengan en las dinámicas generadas por actores educativos.

Otra variable resaltable fue el sentido de pertenencia a la IE. Así, aquellas I.E en las que se evidencia un alto sentido de pertenencia por parte de los estudiantes presentan menores brechas de rendimiento entre niveles socioeconómicos, mientras que las IE con un bajo sentido de pertenencia, en promedio, exponen mayores brechas por nivel socioeconómico. Esto resulta interesante por dos aspectos. Primero, facilita pensar en acciones realizables desde la gestión de la IE; tales acciones deben buscar promover un ambiente que, además de educar, acoja y reconozca al estudiante para así fomentar su sentido de pertenencia hacia la IE. Segundo, tal resultado nos indica que una identidad a nivel colectivo (aula de clase, institución educativa) puede promover sensaciones positivas que se vinculen con un mejor desempeño académico, tema que sería interesante indagar en futuras investigaciones.

A modo de síntesis, los resultados expuestos plantean los siguientes retos para el desarrollo de políticas educativas y desarrollo de programas educativos que promuevan un mejor rendimiento escolar.

- Promover las estrategias de manejo de clima en el aula en la formación docente: Los resultados del presente estudio muestran que las IE que cuentan con docentes que logran reducir las interrupciones por parte de los estudiantes durante el dictado de clase son aquellas que logran mejores resultados de rendimiento con sus estudiantes. Así, es necesario que los centros de formación de docentes (técnica y universitaria) trabajen prácticas docentes que permitan reducir las interrupciones en las aulas de clase por parte de los estudiantes. 
- Promover el abastecimiento de recursos educativos: Los resultados nos muestran que aquellas IE públicas que cuentan con recursos educativos para la enseñanza obtienen buenos resultados de rendimiento educativo. Debido a ello, se debe dirigir el presupuesto hacia la entrega y dotación de recursos educativos en las instituciones.

- Promover el clima escolar en las instituciones educativas: Es necesario que se elaboren estrategias para mejorar el clima escolar en las instituciones educativas. Estos resultados, al igual que los de otros estudios, identifican que buenas relaciones entre docentes y estudiantes generan un sentido de pertenencia en los últimos que disminuye las brechas socioeconómicas de rendimiento en matemáticas.

\section{Agradecimientos}

Los autores agradecen al proyecto de Fortalecimiento de la Gestión de la Educación en el Perú (Forge) por el financiamiento proporcionado para llevar a cabo esta investigación.

\section{Nota biográfica}

\section{JUAN LEÓN}

Es candidato a PhD en Teorías y Políticas Educativas y Educación Internacional Comparada por la Universidad Estatal de Pensilvania. Bachiller en Ciencias Sociales con mención en Economía por la Pontificia Universidad Católica del Perú. Actualmente, es investigador asociado de Grade del área Educación y aprendizajes, y profesor de la Escuela Académico Profesional de Psicología de la Universidad Antonio Ruiz de Montoya. Sus temas de investigación abarcan los efectos escolares, la eficacia escolar, los efectos de la escolaridad, la educación bilingüe, las inequidades educativas, el desarrollo infantil y la evaluación de impacto.

\section{Min-Jong Youn}

Es PhD en Teorías y Políticas Educativas y Educación Internacional Comparada por la Universidad Estatal de Pensilvania. Es magíster en Sociología de la Educación y licenciado en Sociología por la Universidad de Chung-Ang en Seúl, Corea del Sur. Actualmente, es investigador asociado en el área de Investigación y Planificación de Presupuesto del Instituto Nacional de Políticas para la Juventud en Corea del Sur. Sus temas de investigación abarcan los efectos escolares, las inequidades educativas, el desarrollo infantil, la movilidad social y la teoría social. 


\section{Referencias}

Agüero, J. \& Cueto, S. (2004). Dime con quién estudias y te diré cómo rindes: Peer-effects como determinantes del rendimiento escolar. Lima: Consorcio de Investigación Económica y Social.

Albernaz, Â., Ferreira, F. H., \& Franco, C. (2002). Qualidade e eqüidade na educação fundamental brasileira. Pesquisa e Planejamento Económico, 32(3).

Asmad, U., Palomino, D., Tam, M. \& Zambrano, G. (2004). Una aproximación a la alfabetización matemática de los estudiantes peruanos de 15 años: Resultados del Perú en la evaluación internacional PISA (Documento de Trabajo 10). Lima: UMC-Ministerio de Educación

Banco Mundial (1999). Peru Education at a Crossroads. Challenges and opportunities for the 21st century - Vol. I (Report No.19066-PE). Washington, DC: The World Bank.

Benavides, M. (2002). Para explicar las diferencias en el rendimiento en matemáticas de cuarto grado en el Perú urbano: análisis de resultados en base a un modelo básico. En J. Rodríguez \& S. Vargas (Eds.), Análisis y resultados y metodología de las pruebas Crecer 1998 (pp. 93-107). Lima: Ministerio de Educación; Programa Mecep, Documento de trabajo 13.

Benavides, M.; Leon, J. \& Etesse, M. (2014). Desigualdades educativas y segregación en el sistema educativo peruano. Una mirada comparativa de las pruebas Pisa 2000 y 2009. Lima: Grade.

Branch, R. M. \& Deissler, C. H. (2013). Capítulo 7: Processess. En A. Januszewski \& M. Molenda (Eds.), Educational technology: A definition with commentary. Nueva York: Routledge.

Carroll, J.B. (1963). A model of school learning. Teachers College Record, 64, 722-733.

Coleman, J. S., Campbell, E. Q., Hobson, C. J., McPartland, J., Mood, A. M., Weinfeld, F. D. \& York, R. L. (1966). Equality of Educational Opportunity. Washington, DC: U.S. Government Printing Office.

Caro, D. (Coord.) (2004). Una aproximación a la alfabetización lectora de los estudiantes peruanos de 15 años. Resultados del Perú en la evaluación internacional Pisa (Documento de trabajo $\mathrm{N}^{\circ}$ 6). Lima: Unidad de Medición de la Calidad Educativa del Ministerio de Educación.

Cervini, R. (2001). Efecto de la «Oportunidad de aprender» sobre el logro en matemáticas en la educación básica argentina. Revista Electrónica de Investigación Educativa, 3(2). Recuperado de http://77redie.ens.uabc.mx/ vol3no2/contenido-cervini.html

Creemers, B. P. M. (1994). The effective classroom. Londres: Cassell.

Cueto, S. (2004). Factores predictivos del rendimiento escolar, deserción e ingreso a educación secundaria en una muestra de estudiantes de zonas 
rurales del Perú. Education Policy Analysis Archives, 12(35). Recuperado de http://epaa.asu.edu/epaa/v12n35/

Cueto, S., Ramírez, C., León, J. \& Guerrero, G. (2004). Oportunidades de aprendizaje y rendimiento en matemática de los estudiantes de tercero y cuarto grados de primaria en Lima y Ayacucho. En M. Benavides (Ed.), Educación, procesos pedagógicos y equidad: Cuatro Mimeos de investigación (pp. 15-67). Lima: Grade.

Cueto, S., Ramírez, C. \& León, J. (2006). Opportunities to learn and achievement in mathematics in a sample of sixth grade students in Lima, Peru. Educational Studies in Mathematics, 62(1), 25-55.

Cueto, S., Guerrero, G., León, J., Zapata, M. \& Freire, S. (2014). The relationship between socioeconomic status at age one, opportunities to learn and achievement in mathematics in fourth grade in Peru. Oxford Review of Education. doi: 10.1080/03054985.2013.873525

Franco, C., Ortigão, M. I. R., Albernaz, A., Bonamino, A. M. C., Aguiar, G., Alves, F., \& Satyro, N. (2007). Qualidade e equidade em educação: reconsiderando o significado de «fatores intra-escolares». Ensaio. Avaliação e Políticas Públicas em Educação, 15, 277-297.

Goldstein, H. (2003). Multilevel statistical models (3a ed.). Londres: Hodder Arnold Publishing.

Greenwald, R., Hedges, L. \& Laine, R. (1996). The effects of school resources on student achievement. Review of Educational Research, 66(3), 361-396.

Guadalupe, C., León, J. \& Cueto, S. (2013). Charting progress in learning outcomes in Peru using national assessments (Background paper). París: EFA Global Monitoring Report.

Hanushek, E. A. (1986). The economics of schooling: Production and efficiency in public schools. Journal of Economic Literature, 24(3), 1141-1177.

Jencks, C. S., Smith, M., Acland, H., Bane, M. J., Cohen, D., Gintis, H., Heyns, B. \& Michelson, S. (1972). Inequality: A reassessment of the effects of family and schooling in America. New York: Basic Books.

Kline, R. B. (2005). Principles and practice of structural equation modeling (2a ed.). New York: Guilford.

Lee, V. E., Smith, J. B. \& Croninger, R. G. (1997). How High School Organization Influences the Equitable Distribution of Learning in Mathematics and Science. Sociology of Education, 70(2), 129-52.

Lee, V. E. \& Bryk, A. S. (1989). A Multilevel Model of the Social Distribution of High School Achievement. Sociology of Education, 62(3), 172-92.

Lee, V. E., Franco, C. \& Albernaz, A. (2009). Quality and equality in Brazilian secondary schools: A multilevel cross-national school effects study. International Review of Comparative Sociology, 1(1), 25-61. 
Laboratorio Latinoamericano de Evaluación de la Calidad de la Educación [Llece] (2001). Primer estudio internacional comparativo sobre Lenguaje, Matemática y factores asociados para alumnos del tercer y cuarto grado de la educación básica. Santiago de Chile: Unesco.

Laboratorio Latinoamericano de Evaluación de la Calidad de la Educación [Llece] (2002). Estudio cualitativo de las escuelas con resultados destacables en siete países Latinoamericanos. Santiago de Chile: Unesco.

Laboratorio Latinoamericano de Evaluación de la Calidad de la Educación [Llece] (2010). Factores asociados al logro cognitivo de los estudiantes de América Latina y el Caribe. Santiago de Chile: Unesco.

Luyten, H., Scheerens, J. \& Van Ravens, J. (Eds.) (2011). Perspectives on educational quality: Illustrative outcomes on primary and secondary schooling in the Netherlands (Vol. 1). Londres: Springer Science \& Business Media.

Miranda, L. (2008). Factores asociados al rendimiento escolar y sus implicancias para la política educativa del Perú. Análisis de programas, procesos y resultados educativos en el Perú. En M. Benavides (Ed.), Análisis de programas, procesos y resultados educativos en el Perú. Contribuciones empíricas para el debate. Lima: Grade.

Murillo, F. J. (2003). Una panorámica de la investigación iberoamericana sobre eficacia escolar. Revista Electrónica Iberoamericana sobre Calidad, Eficacia y Cambio en Educación, 1(1).

Murillo, F. J., Castañeda, E., Cueto, S., Donoso, J. M., Fabara, E., \& Hernández, M. L. (2007). Investigación iberoamericana sobre eficacia escolar. Bogotá: Convenio Andrés Bello.

Organisation for Economic Co-operation and Development [OECD] (2010). Pisa 2009 Results: "Overcoming social background. Equity in learning opportunities and outcomes» (Vol. II). Recuperado de http://dx.doi. org/10.1787/9789264091450-en.

(2013). PISA 2012 Results: «Pisa 2012 results: Excellence through equity: Giving every student the chance to succeed» (Vol. II). Recuperado de http://www.oecd.org/pisa/keyfindings/pisa-2012results-volume-ii.htm

(2014). PISA 2012 Technical Report. Recuperado de https://www.oecd.org/pisa/pisaproducts/PISA-2012-technical-reportfinal.pdf

oecd.org/pisa/

(s.f.). PISA. What's new. OECD. Recuperado de www.

Piñeros, L. J. \& Rodríguez Pinzón, A. (1998). Los insumos escolares en la educación secundaria y su efecto sobre el rendimiento académico de los estudiantes: Un estudio en Colombia. Washington, D.C.: Banco Mundial. 
Plowden Committee (1967) Children and their primary schools. Londres: HMSO.

Raudenbush, S. W. \& Bryk, A. S. (2002). Hierarchical linear models: Applications and data analysis methods ( $2^{\mathrm{a}}$ ed.). Newbury Park, CA: Sage.

Scheerens, J. (1990). School effectiveness research and the development of process indicators of school functioning. School Effectiveness and School Improvement, 1, 61-80. Lisse: Zwets \& Zeitlinger.

Scheerens, J. \& Bosker, R. (1997). The foundations of educational effectiveness. Oxford: Pergamon Press.

Schmelkes, S., Martínez, F. Noriega, M. C. \& Lavín, S. (1996). The quality of primary education in Mexico: A study of five zones. París: International Institute for Educational Planning.

Stringfield, S. C. \& Slavin, R. E. (1991). A hierarchical longitudinal model for elementary school effects. En B. P. M. Creemers \& G. J. Reezigt (Eds.), Evaluation of educational effectiveness. Groningen: ICO.

Unidad de Medición de la Calidad [UMC] \& Grade (2001). El Perú en el primer estudio internacional comparativo de la Unesco sobre lenguaje, matemática y factores asociados en tercer y cuarto grado. Boletín UMC 9. Lima: Ministerio de Educación.

UMC (2004). Una aproximación a la alfabetización matemática y científica de los estudiantes peruanos de 15 años. Resultados del Perú en la evaluación internacional PISA (Documento de Trabajo 10). Lima: UMC-Ministerio de Educación.

(2006). ¿Cómo disminuir la inequidad del sistema educativo peruano y mejorar el rendimiento de sus estudiantes? Factores explicativos más relevantes en la Evaluación Nacional 2004 (Documento de Trabajo 21). Lima: UMC-Ministerio de Educación.

Valdivia, M. (2004). La escuela y el rendimiento escolar de los niños en el Perú: Sobre cómo la heterogeneidad condiciona el proceso de descentralización (Draft). Lima: Grade

Walberg, H. J. (1984). Improving the productivity of America's schools. Educational Leadership, 41(8), 19-27. 